\title{
Pearls and Tips in Shoulder Arthroplasty
}

\author{
John W. Sperling, MD \\ Department of Orthopedic Surgery, Mayo Clinic, Rochester, MN, USA
}

\begin{abstract}
There has been rapid evolution in new techniques and technology in shoulder arthroplasty. This has improved the efficiency of the procedures as well as expanded our ability to manage some of the most challenging cases. This article will review key aspects of patient evaluation, discuss key technical steps in primary anatomic and reverse arthroplasty, and lastly provide tips to facilitate revision surgery.

Keywords: Arthroplasty, Replacement, Shoulder
\end{abstract}

There has been a dramatic growth in shoulder arthroplasty worldwide over the past 10 years. An organized approach to the preoperative evaluation and careful attention to key technical steps can improve patient outcomes and minimize complications in both the primary and revision setting.

\section{PREOPERATIVE EVALUATION}

\section{History}

It is important to understand the primary complaint of the patient-is it pain, weakness, or loss of motion? Additionally, one should inquire about the patient's occupation and recreational activities. This will help with understanding the demands the patient will place on the shoulder replacement.

A comprehensive understanding of prior treatment is important including injections, therapy, and prior surgery. Understanding the specific type of prior surgery is important including a history of prior shoulder stabilizations for shoulder instability that can significantly distort the anatomy. It is important to recognize if the patient had any prior problems with wound heal-

Received April 8, 2019; Accepted April 25, 2019

Correspondence to: John W. Sperling, MD

Department of Orthopedic Surgery, Mayo Clinic, 200 First Street S.W.,

Rochester, MN 55902, USA

Tel: +1-507-284-2511

E-mail: sperling.john@mayo.edu ing after prior surgery. A patient may recall a history of a "stitch abscess" or use of antibiotics after prior surgery. This should alert the surgeon about potential low-grade infection. In patients with a history of inflammatory arthritis it is useful to know if other joints are involved or have been replaced including the elbow. It is also essential to get a general sense of the emotional profile of the patient and their ability to conform with postoperative rehabilitation and restrictions following the shoulder replacement.

\section{Physical Examination}

A systematic examination includes attention to the cervical spine and a neurovascular examination. In patients with history of prior surgery, inspection can reveal muscle atrophy and deltoid deficiency. One examines for shoulder range of motion including active and passive abduction, external rotation, and internal rotation. Strength is tested in flexion, abduction, external rotation, as well as internal rotation.

Deltoid function is evaluated by checking the anterior, lateral, and posterior heads of the deltoid muscle. There are certain physical examination signs that are helpful to note including the Gerber lift off sign and abdominal compression test to assess for subscapularis function and the Hornblower sign for the status of the posterior rotator cuff.

\section{Preoperative Imaging}

Plain radiographs should include at minimum an anteriorposterior (AP) view in external rotation, AP view in inter- 
Sperling. Pearls and Tips in Shoulder Arthroplasty

Clinics in Orthopedic Surgery • Vol. 11, No. 3, 2019• www.ecios.org

nal rotation, axillary view, and a Neer outlet view. On the AP radiographs, one evaluates the medial-lateral acromiohumeral distance for evidence of glenoid erosion and the superior-inferior acromiohumeral distance for evidence of possible rotator cuff insufficiency. One can get a general sense of humeral bone stock by examining the diameter of the humeral canal, thickness of the cortices, and bone density. Lastly, one can look for associated acromial wear. On the axillary view, one can assess for glenoid version, bone loss, and subluxation. The outlet view can assist with understanding the acromial morphology.

A computed tomography (CT) scan has become standard in many surgeons practice to facilitate preoperative planning. Custom patient guides are available from a wide variety of manufacturers to assist with planning and intraoperative placement of components. ${ }^{1-3)}$ Some surgeons also prefer to have an magnetic resonance imaging to evaluate the soft tissue.

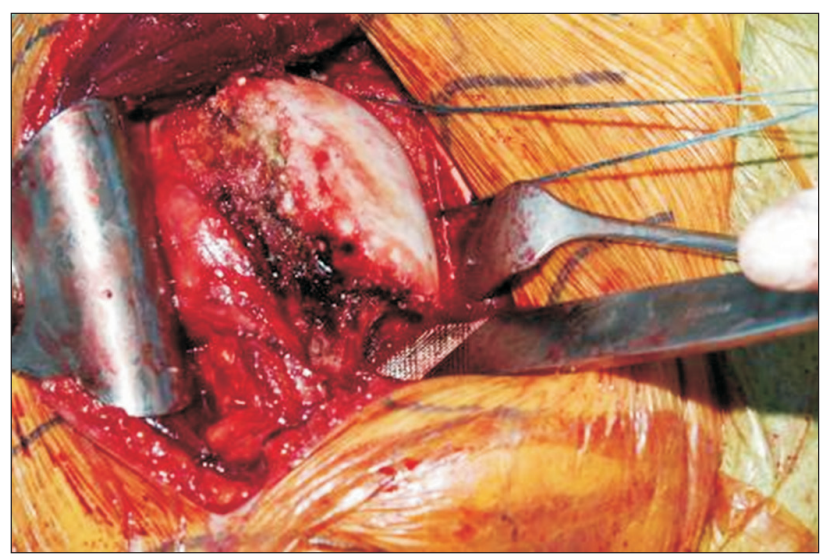

Fig. 1. Inferior capsular release along the anterior and inferior portion of the proximal humerus.

\section{PRIMARY ANATOMIC SHOULDER ARTHROPLASTY: TIPS AND TRICIKS}

The deltopectoral approach is the standard approach for anatomic shoulder arthroplasty. It is easiest to find the deltopectoral interval proximally and there is usually a small triangle of fat present between the pectoralis and deltoid in this location. The cephalic vein is typically left medially although many surgeons prefer to retract it laterally. It is helpful to clear adhesions in the subacromial as well as the subdeltoid space.

There is significant debate in regard to the best management of the subscapularis in anatomic shoulder arthroplasty. ${ }^{4,5)}$ It is the author's preference to perform a tenotomy of the subscapularis. If one tenodesis the long head of the biceps proximally within the groove, one can then use the bicipital sheath and the biceps tendon to reinforce the subscapularis repair at the conclusion of the procedure.

A large inferior capsular release off the humerus is essential for glenoid exposure as well as to improve the patient's overhead motion. This is done with electrocautery and staying directly on bone with progressive external rotation of the arm in the adducted position. The release is typically extended to the 4 oclock position (Fig. 1).

A Browne retractor is then used to expose the proximal humerus and allow direct entry into the superior part of the humerus. With a stemmed prosthesis, a burr is then used to create a generous entry hole in the proximal humerus. Circular reamers are then used in a nonaggressive manner. The rate of isolated humeral loosening is rare; therefore, the surgeon doesn't need to force in a tight humeral stem. The author prefers to cut the humeral head in $30^{\circ}$ of retroversion for both anatomic and reverse arthroplasty.

Stemless shoulder arthroplasty has a number of potential benefits including bone preservation and the potential for future conversion to reverse arthroplasty.
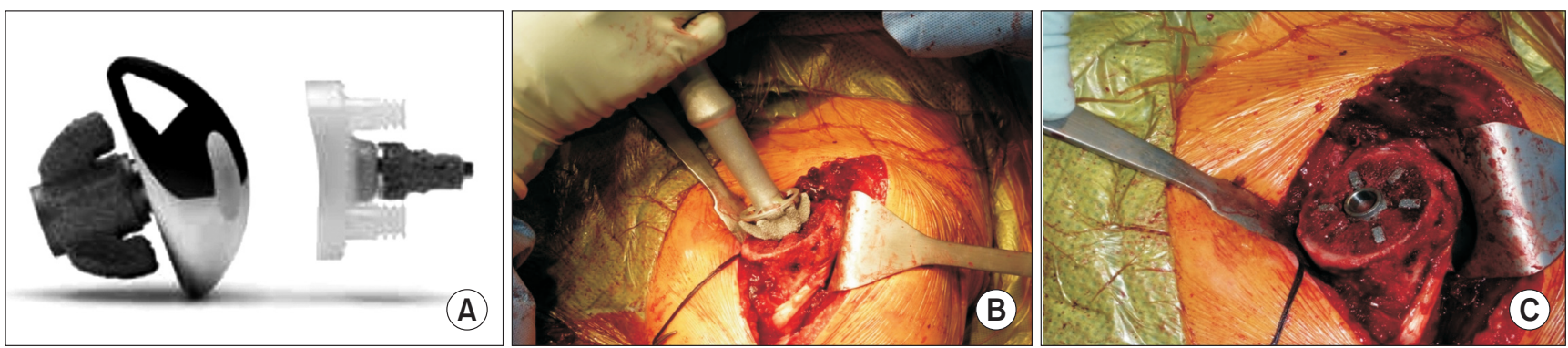

Fig. 2. Stemless shoulder replacement with porous ingrowth metal and a hybrid glenoid component. (A) Nano stemless implant. (B) Implanation of stemless humeral component. (C) Seating of the implant. 
Sperling. Pearls and Tips in Shoulder Arthroplasty

Clinics in Orthopedic Surgery • Vol. 11, No. 3, 2019• www.ecios.org

The author prefers a stemless component with ingrowth metal that is available in a large number of sizes (Fig. 2). Additionally, the ability to dial the humeral head in the anatomic position is very important to maximize function and minimize rotator cuff failure.

In the author's experience there are four common reasons for difficult glenoid exposure: (1) lack of deltoid mobilization, (2) insufficient capsular release, (3) too high a humeral head cut, and (4) insufficient osteophyte removal. For anatomic shoulder arthroplasty, the author prefers a hybrid glenoid with cemented peripheral polyethylene pegs and a central post with ingrowth metal for long-term fixation. In contrast, the history of fully metal backed glenoid components has been poor overall with accelerated polyethylene wear, instability, and high rates of failure. Moreover, removal of a fully metal backed glenoid component can be extremely challenging. Therefore, the author prefers a hybrid glenoid that has the benefits of long-term bone ingrowth and the ease of revision if necessary in the future.

\section{NEW TECHNIQUES IN PRIMARY REVERSE ARTHROPLASTY: BONE PRESERVING BASEPLATE}

There has been evolution of new techniques in reverse arthroplasty that can significantly preserve glenoid bone. The average glenoid has $7^{\circ}$ to $11^{\circ}$ of superior inclination. Creating inferior tilt has been shown to be extremely important to prevent baseplate loosening. ${ }^{6}$ Inferior tilt is traditionally created by reaming away central and inferior glenoid bone. Rather than reaming away this central and inferior bone, in a new bone preserving technique, an augmented baseplate is used to create the tilt. Benefits of this technique include (1) less bone removal, (2) longer central and peripheral screws, (3) seating on hard subchondral bone not soft cancellous bone, (4) lateralization with improved tension on the deltoid and remaining rotator cuff, (5) decreased scapular notching, and (6) less risk of greater tuberosity and acromial impingement (Fig. 3).

In those cases with bone deficiency, an augment can be used rather than bone graft. Challenges with bone graft include resorption, fragmentation during insertion, and lack of local bone in revision surgery. Additionally, if the bone graft is slightly too thick, the baseplate cannot be
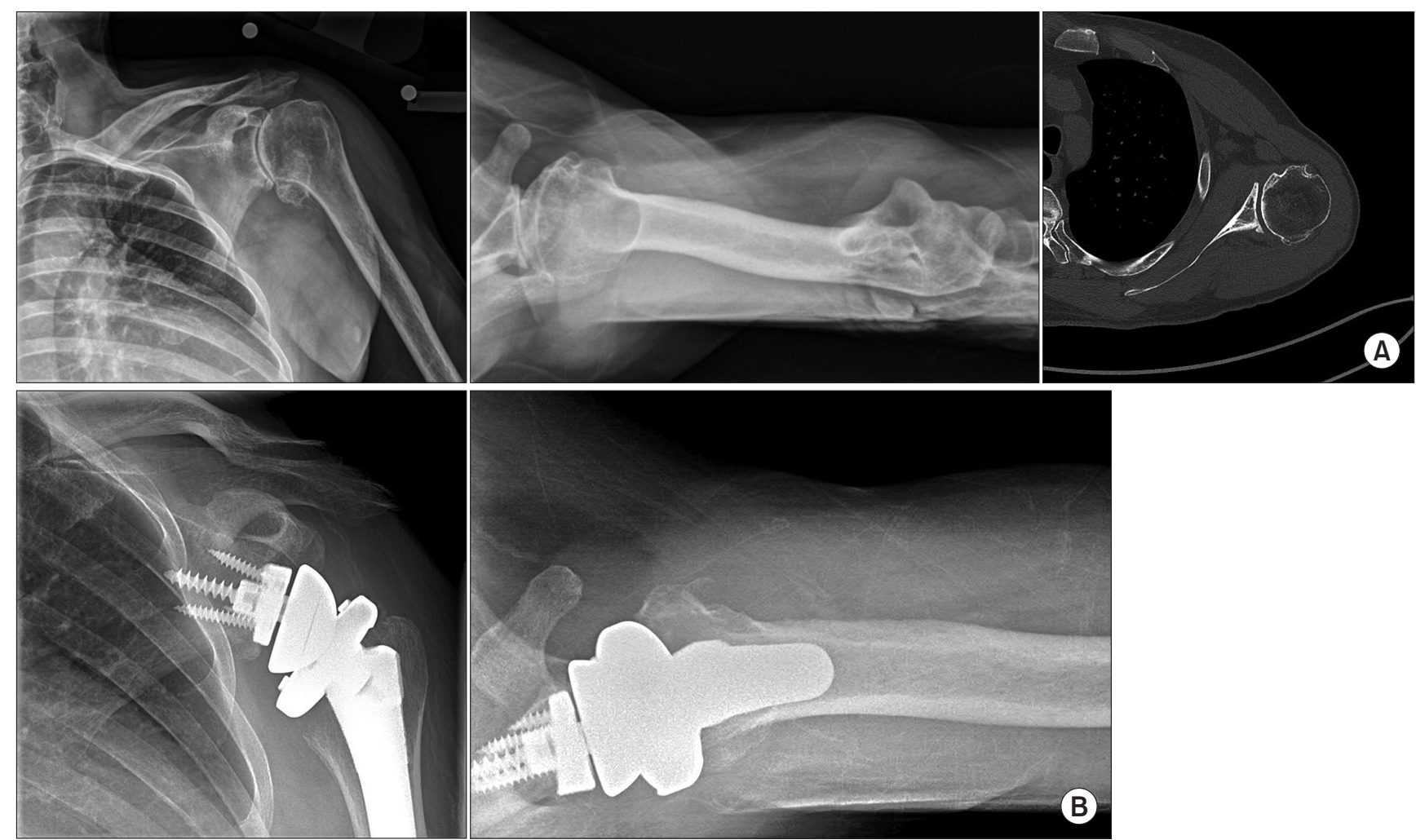

Fig. 3. (A) Significant posterior glenoid erosion. (B) An augmented baseplate is used posteriorly to help make up for the missing bone. 
seated on native bone. Moreover, if the rotation of the graft or the baseplate is not correct, there will be a gap behind the baseplate and incomplete seating of the component. Augments have been used for many years in hip and knee reconstruction due to a wide variety of sizes as well as precise preparation and placement. These are becoming more commonly used in shoulder reconstruction as well.

\section{REVISION SURGERY TIPS AND TRICKS}

\section{Glenoid Component Loosening}

The surgeon may consider revision to a hemiarthroplasty with bone grafting in certain patients with glenoid compo- nent loosening. While the procedure is technically easier to perform than reverse arthroplasty, the pain relief and function is not as predictable. The surgeon may consider revision to anatomic shoulder arthroplasty if the rotator is intact; however, solid glenoid fixation can be difficult to obtain. Therefore, the mainstay treatment for glenoid loosening has become revision to a reverse total shoulder arthroplasty (TSA). ${ }^{7}$

Exposure in these cases may be challenging, particularly with severe glenoid bone loss. Technical pearls include performing a large inferior capsular release off the humerus. It is helpful to have a clear process for component removal. A router bit is initially used circumferen-
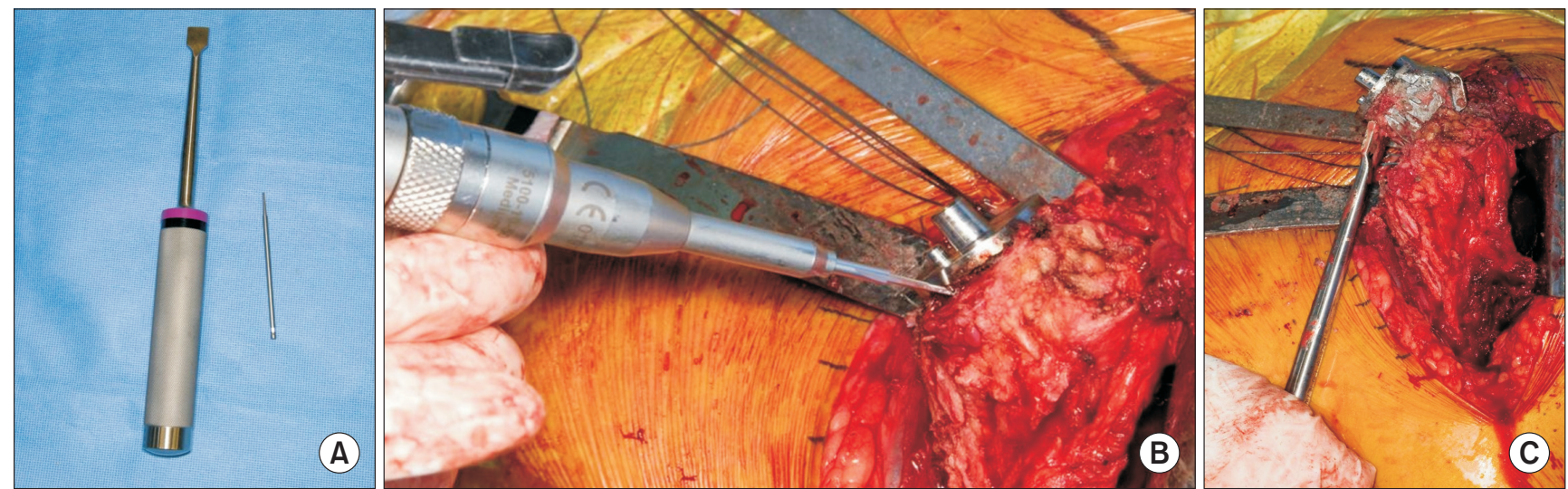

Fig. 4. (A) A router bit and a square tip impactor. (B) The router bit is used circumferentially around the proximal humerus. (C) The square tip impactor is then used against the collar of the implant.

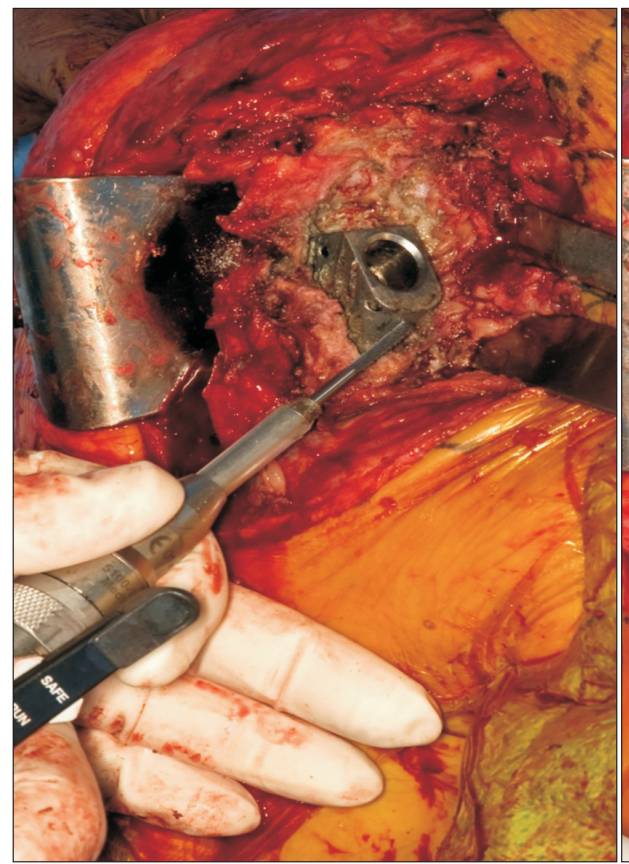

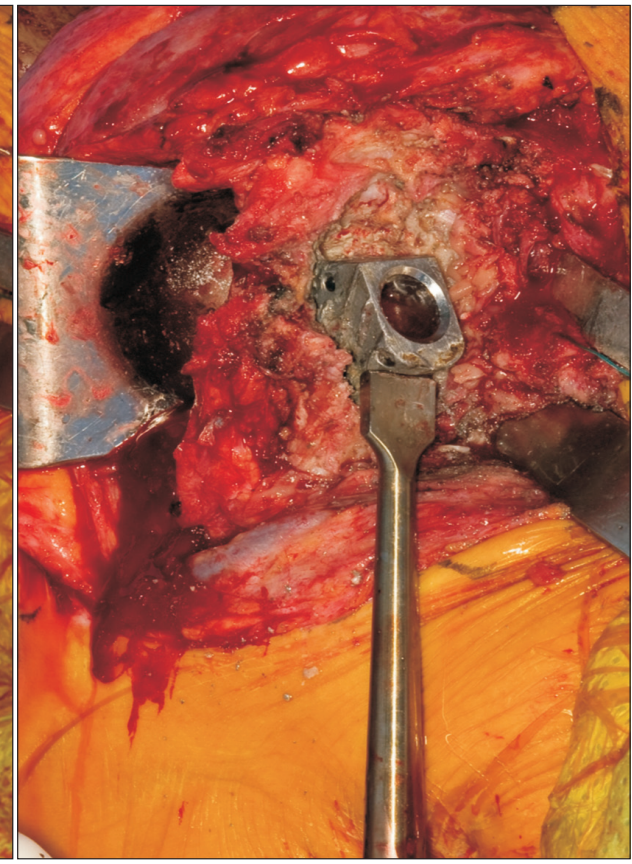

Fig. 5. Helicoidal bur is used to create a ledge in an implant without a collar. The square tip impactor is then used to remove the stem. 
tially around the proximal humerus (Fig. 4). A square tip impactor is then used from below with a mallet to remove the humeral component. When the humeral component does not have a collar, one can make a collar with a helicoidal bur (Fig. 5). There is good evidence that one can use short stems in revision to reverse arthroplasty as well as a cement within cement technique rather than bypassing the old pedestal with a long stem. ${ }^{8,9)}$ This can greatly facilitate the current and future revision surgery.

In cases with prior humeral fracture, tumor surgery, or revision shoulder arthroplasty, there may be significant humeral bone loss. One can use allograft to make up the missing humeral bone. However, graft resorption as well as graft availability is a concern. In hip and knee arthroplasty, metal has become the standard to make up for missing bone. There are now a variety of choices for the shoulder to make up for the missing bone with metal (Fig. 6). This allows for precise instrumentation as well as component placement. If there has not been prior use of cement in the humerus, one may consider not cementing the humeral component. However, in those revision cases where there has been the use of a prior cemented humeral stem, because of the increased stress placed on the component as one moves lower down the humeral shaft, the humeral
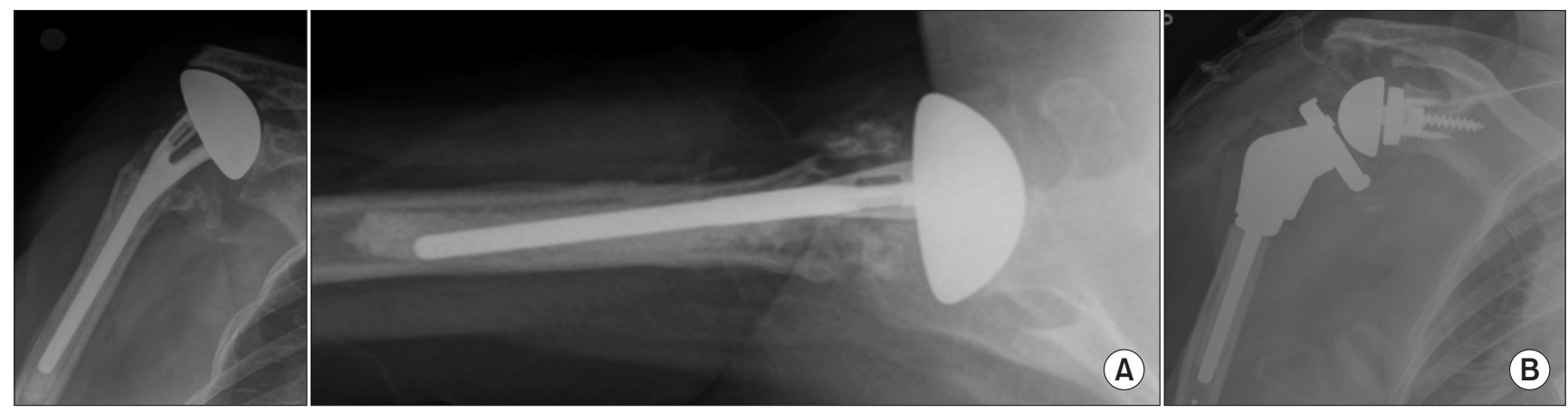

Fig. 6. (A) A hemiarthroplasty for fracture with tuberosity resorption and proximal humeral bone deficiency. (B) A metal implant is used to recreate tension on the deltoid.
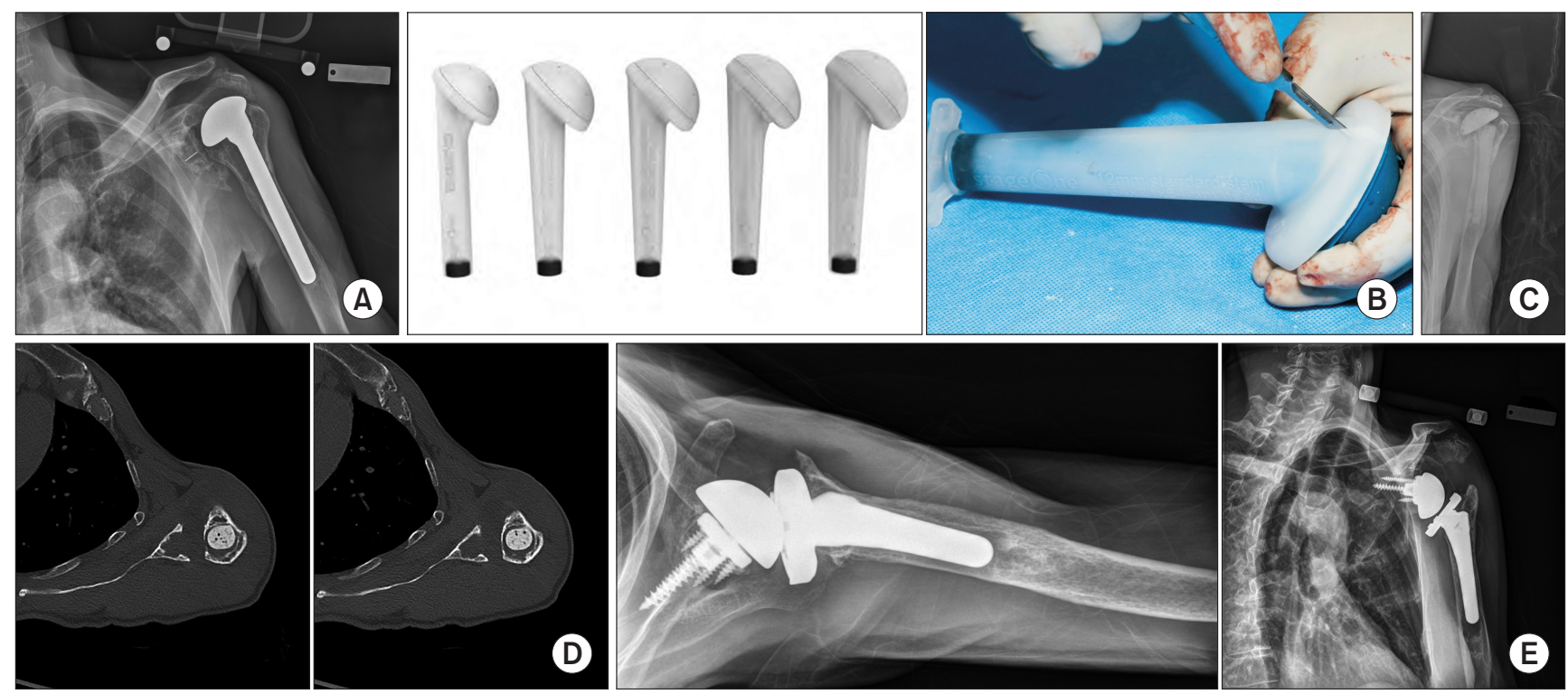

Fig. 7. (A) An infected shoulder arthroplasty with a loose humeral component that has shifted in position. (B) An antibiotic spacer can be templated ahead of the case. This facilitates the flow of surgery and allows the surgeon to titrate the antibiotics for the individual patient. (C) Radiograph with the cement spacer in place. (D) A computed tomography scan shows the bone remaining medially. This is ideally suited for central screw fixation. (E) A baseplate with a modular central screw engages the far cortex and provides compression. The glenoid baseplate is placed in the best bone and then the glenosphere is "dialed" for glenoid coverage. Additionally, a shorter humeral stem is used and the old pedestal is not bypassed. 
component is typically cemented.

\section{Glenoid Arthritis}

Glenoid arthritis is characterized by progressive shoulder pain in a patient with a hemiarthroplasty. The radiographs regularly show progressive glenoid bone loss. A CT scan can be useful for preoperative planning. An ultrasound may also be helpful to assess the status of the rotator cuff.

Revision to an anatomic TSA by implantation of a glenoid component can be performed if there is adequate glenoid bone stock and an intact rotator cuff. However, more commonly, there is insufficiency of glenoid bone stock or accompanying rotator cuff tearing. Therefore, reverse arthroplasty is being progressively used. ${ }^{10)}$ The pain relief is quite expected. However, the range of motion is frequently less than an uncomplicated primary TSA.

\section{Infection}

The diagnosis of infection may be easy to make in cases with frank drainage; however, the large majority of time it is challenging to establish. Preoperative tests such as complete blood count, sedimentation rate and C-reactive protein are usually normal. Culture of aspirated fluid is also difficult because fluid may not be able to be obtained and is usually normal. Intraoperative pathology is also usually normal. Intraoperative cultures are the gold standard. One should consider taking between three and five samples. There is some increased discussion of a one stage procedure; however, the most commonly performed for chronic infection is a two stage procedure. This is relatively successful in eradicating infection (Fig. 7). ${ }^{11,12)}$ However, function is oftentimes compromised with elevated morbidity and cost.

\section{Rotator Cuff Failure and Instability}

The majority of patients with shoulder instability after anatomic shoulder arthroplasty have both soft tissue imbalance and component malposition. Rotator cuff repair after shoulder arthroplasty has also had a poor success rate. Additionally, revision surgery with anatomic components and soft tissue reconstruction has had a high failure rate. Consequently, both instability and symptomatic rotator cuff dysfunction after anatomic shoulder arthroplasty are preferably treated with revision to a reverse prosthesis (Fig. $8){ }^{13)}$
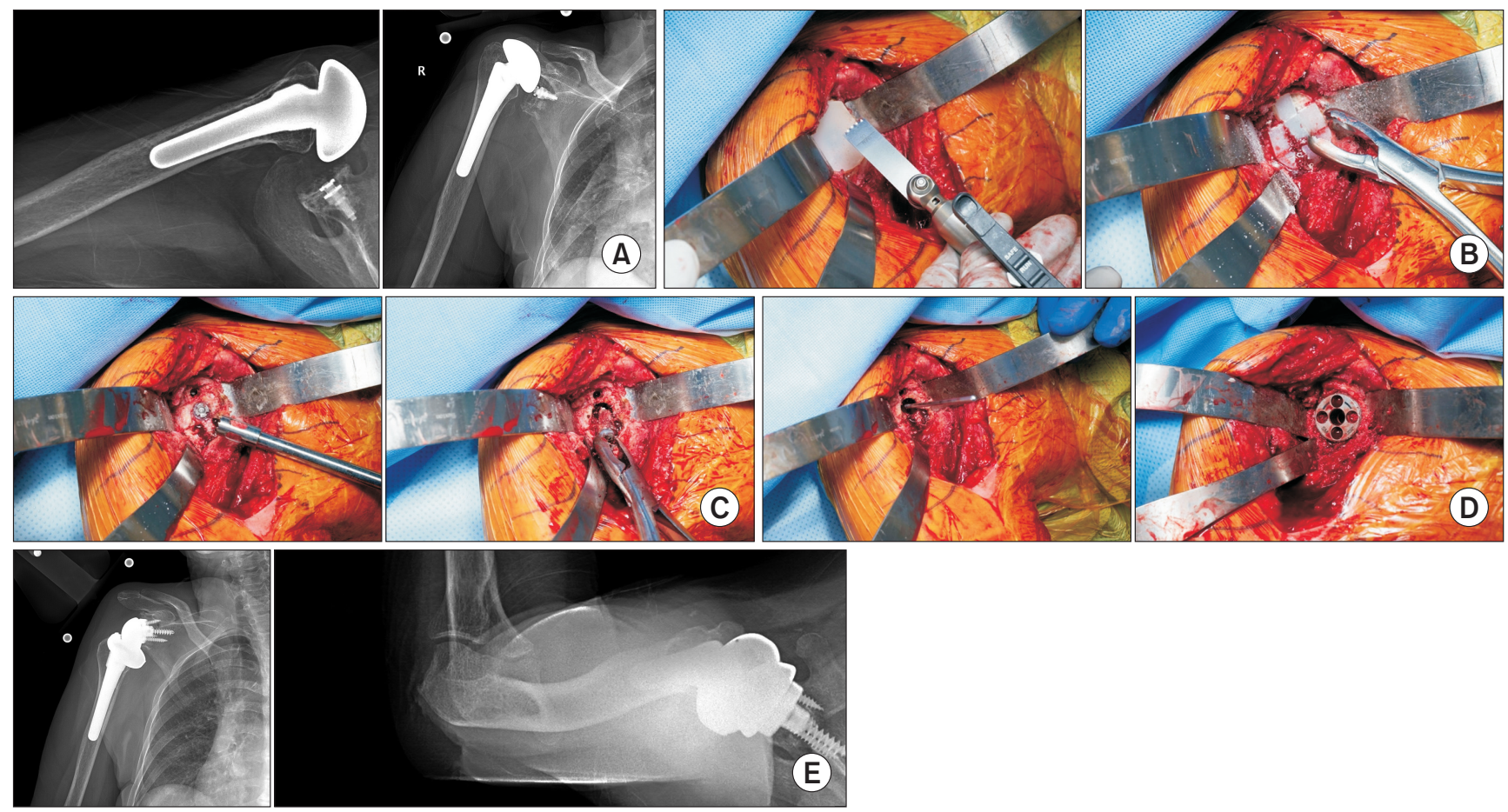

Fig. 8. (A) A patient who underwent repair of a large rotator cuff tear at the time of anatomic shoulder arthroplasty at an outside institution. She has a large rotator cuff tear, anterior subluxation of the humeral head, and the inability to raise her arm. (B) The glenoid component is split with a saw and removed with two horizontal cuts and two vertical cuts. (C) The central ingrowth post is removed. (D) An augmented baseplate is used to create tilt rather than reaming away a large amount of central and inferior bone. (E) Postoperative imaging revealing the convertible stem and augmented baseplate used to create the tilt. 
Sperling. Pearls and Tips in Shoulder Arthroplasty

Clinics in Orthopedic Surgery • Vol. 11, No. 3, $2019 \bullet$ www.ecios.org

\section{CONCLUSIONS}

Shoulder arthroplasty has evolved into a durable procedure for managing a breadth of complex pathologies. A detailed and careful preoperative evaluation together with key technical steps can improve outcomes and minimize complications.

\section{CONFLICT OF INTEREST}

No potential conflict of interest relevant to this article was reported.

\section{REFERENCES}

1. Amini MH, Ricchetti ET, Iannotti JP. Three-dimensional templating and use of standard instrumentation in primary anatomic total shoulder arthroplasty. JBJS Essent Surg Tech. 2017;7(3):e28.

2. Ricchetti ET, Jun BJ, Cain RA, et al. Sequential 3-dimensional computed tomography analysis of implant position following total shoulder arthroplasty. J Shoulder Elbow Surg. 2018;27(6):983-92.

3. Iannotti JP, Weiner S, Rodriguez E, et al. Three-dimensional imaging and templating improve glenoid implant positioning. J Bone Joint Surg Am. 2015;97(8):651-8.

4. Lapner PL, Wood KS, Zhang T, Athwal GS. The return of subscapularis strength after shoulder arthroplasty. J Shoulder Elbow Surg. 2015;24(2):223-8.

5. Sacevich N, Athwal GS, Lapner P. Subscapularis management in total shoulder arthroplasty. J Hand Surg Am. 2015;40(5):1009-11.

6. Gutierrez S, Greiwe RM, Frankle MA, Siegal S, Lee WE 3rd. Biomechanical comparison of component position and hardware failure in the reverse shoulder prosthesis. J Shoulder Elbow Surg. 2007;16(3 Suppl):S9-12.

7. Alentorn-Geli E, Clark NJ, Assenmacher AT, et al. What are the complications, survival, and outcomes after revision to reverse shoulder arthroplasty in patients older than 80 years? Clin Orthop Relat Res. 2017;475(11):2744-51.
8. Wagner ER, Statz JM, Houdek MT, Cofield RH, SanchezSotelo J, Sperling JW. Use of a shorter humeral stem in revision reverse shoulder arthroplasty. J Shoulder Elbow Surg. 2017;26(8):1454-61.

9. Wagner ER, Houdek MT, Hernandez NM, Cofield RH, Sanchez-Sotelo J, Sperling JW. Cement-within-cement technique in revision reverse shoulder arthroplasty. J Shoulder Elbow Surg. 2017;26(8):1448-53.

10. Merolla G, Wagner E, Sperling JW, Paladini P, Fabbri E, Porcellini G. Revision of failed shoulder hemiarthroplasty to reverse total arthroplasty: analysis of 157 revision implants. J Shoulder Elbow Surg. 2018;27(1):75-81.

11. Baghdadi YM, Maradit-Kremers H, Dennison T, et al. The hospital cost of two-stage reimplantation for deep infection after shoulder arthroplasty. JSES Open Access. 2017;1(1):158.

12. Assenmacher AT, Alentorn-Geli E, Dennison T, et al. Two-stage reimplantation for the treatment of deep infection after shoulder arthroplasty. J Shoulder Elbow Surg. 2017;26(11):1978-83.

13. Hernandez NM, Chalmers BP, Wagner ER, Sperling JW, Cofield RH, Sanchez-Sotelo J. Revision to reverse total shoulder arthroplasty restores stability for patients with unstable shoulder prostheses. Clin Orthop Relat Res. 2017;475(11):2716-22. 